\title{
Editorial
}

J. Synchrotron Rad. (1996). 3, 247

\section{JSR: Required Reading}

\author{
S. Samar Hasnain, ${ }^{a}$ John R. Helliwell ${ }^{b}$ and Hiromichi Kamitsubo ${ }^{c}$ \\ a Synchrotron Radiation Department, CCLRC Daresbury Laboratory, Warrington WA4 4AD, \\ UK, ${ }^{b}$ Department of Chemistry, University of Manchester, Manchester M13 9PL, UK, and \\ 'Jaeri-riken Spring-8 Project Team, Kamigori-cho, Ako-gun, Hyogo 678-12, Japan
}

As we enter our third year and write this editorial, we have received the first major review of the Journal of Synchrotron Radiation (JSR) [Nature (London) (1996), 383, 42]. It is worth repeating some of the points made in this independent review. It says that 'developments in the application of synchrotron radiation research have benefited enormously from fertilization between otherwise distinct research areas, and there is no doubt that this new journal will play an important part in furthering such interdisciplinary interactions'. The review goes on to say that the "speed, together with the quality of the contributions so far and the high standard of production. makes the journal attractive to authors and required reading for workers in what worldwide is still a rapidly expanding field'. We note that the reviewer points out that even though the quality of articles in $J S R$ has been high, the issues have remained fairly slim.

Last November we reiterated our main objectives, namely that $J S R$ should become the focus of the whole of the synchrotron radiation community and to accept papers only of a high standard covering sources, instrumentation, methods and applications. Moreover, we have strived to provide fast and efficient publication to a high technical standard so that the rapidly expanding community is informed of the latest developments quickly and effectively. The review cited above confirms that these objectives are being met. Our main focus now is to increase the size of the issues without compromising the quality of the papers. Even though we are receiving a larger number of quality contributions, as is reflected by the current issue, a number of highly suited papers are being published elsewhere. We would urge you to always consider $J S R$ as the first choice when you have a result, method or an instrumental facility which may be of interest to the rest of the synchrotron radiation community. It is only through your high-quality contributions that our community will be able to sustain the high standards of the journal. $J S R$ offers the opportunity for setting standards and defining performance indicators for cutting-edge instrumentation, vital for achieving the full potential of advanced techniques. We also call upon you to ensure that $J S R$ is available on your library shelves so that new users become aware of the powers of synchrotron radiation.

The six issues of 1996 have covered the whole range of subjects spanning the production of synchrotron radiation and its utilization throughout the spectral region. In each of the issues the interdisciplinary nature of the journal and potential for cross-fertilization has been obvious and the current issue is no exception, where the generation of even more intense beams is reported alongside time-resolved $\mathrm{X}$-ray diffraction of muscle fibres. Several 'first results' from third-generation sources are reported.

The growth of synchrotron radiation has continued worldwide with the news that first beam has been established in the Brazilian synchrotron radiation source. Next year, SPring-8, the world's highest energy synchrotron radiation source, is due to produce its first beam, and first results are expected to be reported at SRI-97, the refereed proceedings of which will be published in JSR. The excitement of continued growth and opportunities can also be felt in new concepts emerging for the fourthgeneration synchrotron radiation sources, whether these are large centralized sources such as X-ray free-electron lasers or distributed 'single-user' customized synchrotron radiation sources. $J S R$ will continue to play its part in providing the necessary platform for rapid communication and scientific and technical exchange necessary for the evolution of these ideas into the synchrotron radiation sources and their applications in the next millennium. 